\title{
Advanced Therapy Medicinal Products: How to Bring Cell-Based Medicinal Products Successfully to the Market - Report from the CAT-DGTI-GSCN Workshop at the DGTI Annual Meeting 2014
}

\author{
Patrick Celis ${ }^{a, b} \quad$ Nicolas Ferry ${ }^{a, c} \quad$ Marit Hystad $^{a, d} \quad$ Martina Schüßler-Lenz ${ }^{a, e}$ \\ Pieter A. Doevendans ${ }^{a, f}$ Egbert Flory ${ }^{a, e}$ Claire Beuneu $^{a, g} \quad$ Ilona Reischl $^{a}$, $h$ \\ Paula Salmikangas ${ }^{a, i}$ \\ ${ }^{a}$ Committee for Advanced Therapies, European Medicines Agency, London, UK; \\ ${ }^{b}$ European Medicines Agency, London, UK; \\ ${ }^{\mathrm{c}}$ Agence nationale de sécurité du médicament et des produits de santé (ANSM), Paris, France; \\ ${ }^{\mathrm{d}}$ Norwegian Medicines Agency, Oslo, Norway; \\ e Paul-Ehrlich-Institut, Federal Institute for Vaccines and Biomedicines, Langen, Germany; \\ ${ }^{f}$ European Society of Cardiology, University Medical Center Utrecht, Department of Cardiology, Utrecht, the Netherlands; \\ ${ }^{9}$ Agence Fédérale des Médicaments et des Produits de Santé, Brussels, Belgium; \\ hBASG/AGES, Vienna, Austria; \\ i Finnish Medicines Agency, Helsinki, Finland
}

\section{Keywords}

Advanced therapy medicinal products - ATMP .

Committee for Advanced Therapies . CAT

\section{Summary}

On September 11, 2014, a workshop entitled 'Advanced Therapy Medicinal Products: How to Bring Cell-Based Medicinal Product Successfully to the Market' was held at the 47th annual meeting of the German Society for Transfusion Medicine and Immunohematology (DGTI), co-organised by the European Medicines Agency (EMA) and the DGTI in collaboration with the German Stem Cell Network (GSCN). The workshop brought together over 160 participants from academia, hospitals, small- or mediumsized enterprise developers and regulators. At the workshop, speakers from EMA, the Committee for Advanced Therapies (CAT), industry and academia addressed the regulatory aspects of development and authorisation of advanced therapy medicinal products (ATMPs), classification of ATMPs and considerations on cell-based therapies for cardiac repair. The open forum discussion session allowed for a direct interaction between ATMP developers and the speakers from EMA and CAT.

\section{Introduction}

Advanced therapy medicinal products (ATMPs) include gene therapy medicinal products, somatic cell therapy medicinal products and tissue-engineered products [1] and can offer groundbreaking new opportunities for treatment of disease and injury. The latter two categories of ATMPs are often referred to as cellbased medicinal products.

Cell-based medicinal products, unlike cell and tissue transplants, undergo additional manipulation during production, resulting in substantial changes in the characteristics of the cells. They can also be used for a different function in the recipient than the original function in the donor. Because of the novelty, complexity and technical specificities, a specific legislation for ATMPs is in place since 2008 [1]. A specialised committee, the Committee for Advanced Therapies (CAT), has been established to review the quality, safety and efficacy of ATMPs prior to marketing authorisation. After authorisation, ATMPs are subject to a strict post-authorisation surveillance programme to ensure the patients' safety.

While only few ATMPs have been authorised so far, there are many products in the pipeline. This is reflected by the volume of pre-authorisation activities in the CAT, i.e., scientific recommen-

\begin{tabular}{ll}
\hline KARGER & () 2015 S. Karger GmbH, Freiburg \\
& $1660-3796 / 15 / 0423-0194 \$ 39.50 / 0$ \\
Fax +497614520714 & \\
Information@Karger.com & Accessible online at: \\
www.karger.com & www.karger.com/tmh
\end{tabular}


dations on ATMP classification, the certification of quality and non-clinical data generated by micro-, small- or medium-sized enterprises (SMEs) for their ATMPs and scientific advice requests for ATMPs [2]. An analysis of the EudraCT database from 2004 to 2010, showing that over 250 different ATMPs are undergoing clinical testing, confirms the active research and development in the field of ATMPs [3].

SMEs and non-commercial operators (from academias, hospitals and non-for-profit organisations) are the main developers of ATMPs [3]. These developers have needs that are different from the big, established pharmaceutical companies, and therefore CAT has established, from very early in its existence, a proactive approach to reach out to SMEs, academias and hospitals. As one of the initiatives, CAT is co-organising scientific workshops with learned societies. The workshop entitled 'Advanced Therapy Medicinal Product - How to Bring Cell-Based Medicinal Products Successfully to the Market', co-organised with the German Society for Transfusion Medicine and Immunohematology (DGTI) and the German Stem Cell Network (GSCN), was the third of such joint meetings.

The workshop aimed to promote the development of ATMPs, raise awareness of the regulatory tools and incentives available and allow for direct interactions between the ATMP developers and the regulators. It was attended by over 160 participants from academias, hospitals, SME-ATMP developers and regulators. In the first three sessions of the workshop, speakers from CAT, European Medicines Agency (EMA), industry and academias presented and discussed the current challenges and latest regulatory development in the field of ATMPs, their views on the demarcation between ATMPs and other therapies [4] and scientific considerations on quality as well as non-clinical and clinical requirements for cell-based therapies developed for cardiac repair. The fourth session was scheduled as an 'Open Forum Discussion with CAT', especially intended to encourage an active dialogue between the participants and the regulators: this turned out to be an very interactive session, with questions asked on how to interact with CAT and how to get support or classification of ATMPs as well as some technical questions arising from the development of a specific ATMP.

\section{Development and Authorisation of Cell-Based ATMPs in the EU}

Cell-based medicinal products (CBMPs) include somatic cell therapy products and tissue-engineered products. They may be derived from the same tissues and cells, however, there intended use is different: somatic cell therapy products deliver their therapeutic action via pharmacological, metabolic or immunological action, where tissue-engineered products are intended for regeneration, repair or replacement of human tissues or organs. As medicinal products the development of new CBMPs has to follow also all other EU legislation applicable for ATMPs. Most important to note is Directive 2004/23/EC and its implementing directives that lay down requirements for procurement, donation and testing of the tissues and cells used as starting material for CBMPs [5]. Furthermore, the technical requirements for all ATMPs are defined in Directive 2009/120/EC [6].

Dr. Salmikangas mentioned in her presentation that the development of new therapies based on tissues and cells is not straightforward and that many challenges have been observed during the past years. CBMPs are complex pharmaceuticals, for which traditional approaches may not be applicable. For administration of these products novel techniques (e.g. catheters) or surgical procedures may be required, and definition of the safe and efficacious dose may not be a simple task. Also the availability of suitable animal models for non-clinical testing may be limited and the characterisation of the product itself may pose problems. Supply of goodquality raw materials for production may also be challenging, and recently a general monograph of the European Pharmacopoeia on the quality and manufacturing of raw materials for production of ATMPs [7] has been established. It is acknowledged that the wide variety of different products, their limitations (e.g. small sample sizes, short shelf lives etc.) and specific risks of ATMPs call for a specifically tailored approach for their development and evaluation. For this, a risk-based approach [8] has been developed to enhance the regulatory flexibility needed for regulation of these products.

In addition to the scientific challenges there are also developer-related challenges as well as socio-economic and legal/regulatory problems hampering the development of CBMPs. Developers of novel ATMPs come from SMEs, hospitals, academias and even from charities [3]. Due to the limited resources and huge workload, a lot of new products are in early clinical studies, but only few proceed to pivotal studies and towards marketing authorisation. As this sector arises from the traditional transplantation/transfusion area, it has been difficult for some stakeholders to recognise and accept the new standards and requirements for CBMPs that look very alike cell/tissue preparations used in hospitals.

There are still different ethical views within member states concerning use of certain cells and tissues for production of CBMPs. Furthermore, there seem to be challenges in getting funding for the large pivotal clinical trials, and the cost of these products, due to e.g. small batch sizes, are higher compared to traditional remedies leading to higher prices. The reimbursement negotiations for first CBMPs have also turned out difficult leading to slow market access and limited use of the new products within the EU.

Clinical trials and requirements therein are within the remit of national authorities, which may lead to extensive workload especially for multisite confirmatory trials requiring simultaneous submission of applications to multiple national authorities. Also different interpretation of the hospital exemption (article 28 of Regulation 1394/2007) has raised concerns of 'second standard' products and has in some cases led even to conflict with the developing CBMP industry. Therefore, it would be of outmost importance to clarify problematic issues in the current legislation, should the ATMP regulation be opened for revision, as proposed by many stakeholders during the public consultation of the European Commission in $2014[9,10]$. 
To overcome the above mentioned challenges, there are multiple procedures and regulatory services available both at EMA and national level. These were presented at the workshop by Dr. Celis.

Two new procedures are put in place for ATMPs, the ATMP classification procedure and the ATMP certification procedure. The ATMP classification procedure allows for ATMP developers to request a scientific recommendation from the CAT on the classification of their product as ATMP. ATMP classification can be applied for early in the product development; it is open to all developers and will give them regulatory certainty. Over 100 products have been classified by CAT, and a summary of the ATMP classifications is published on the ATMP webpage [11]. ATMP certification is a more restricted procedure, in so far that only SME developers of ATMPs can request the CAT to evaluate the quality/manufacturing and, if available, the non-clinical data obtained with their product. This independent scientific review provides the SMEs with scientific certainty and valuable information to further develop their product (in the light of a future marketing authorisation application). The update of this procedure is limited for the moment, with only 5 certification procedures finalised so far.

Scientific advice is a very important tool to support development. This incentive can be requested for early and late development stages, and is open to all applicants. For ATMP developers, a reduced fee is payable. The CAT is routinely involved in scientific advices related to ATMPs, providing input in the work of the Scientific Advice Working Party. Scientific advice is the forum to agree the methodological approaches for quality as well as nonclinical and clinical development.

Last but not least, the EMA is offering possibilities for direct interactions such as briefing meetings with the EMA Innovation Task Force (which is a platform for early dialogue on scientific, regulatory and legal requirement for innovative products) [12], interactions with the EMA SME office and pre-submission meetings for ATMP certification, scientific advice, orphan designation and marketing authorisation. Also, national competent authorities for medicines are providing support to developers via national scientific advices and national innovation offices. The importance of early interactions with the regulatory authorities (national competent authorities and EMA) was stressed.

\section{Demarcation between ATMPs and Other Therapies: the ATMP Classification Procedure}

Classification of ATMPs is an important task of CAT because it has direct impact on the development programme of a medicinal product. Although the classification is not legally binding, it is usual that other regulatory bodies in Europe (EMA committees, European Commission and national authorities for medicines) will follow the scientific recommendation from the CAT.

The ATMP classification is based on the provisions of the ATMP regulation [1] and the definitions of a medicinal product and gene and somatic cell therapy product in the Annex I to Directive 2001/83/EC [6].
One critical issue with tissues and cells used for treatment of patients is the delineation between products not considered ATMPs which are regulated by the Directive on Tissues and Cells [5] and cell- or tissue-based products that are regulated as medicinal products / ATMPs. In some specific situations, such as autologous products manufactured for a specific patient in the hospital, an interpretation of the current legal framework is needed.

Dr. Kleinhenz from Cytori presented the point of view from his company regarding the use of point-of-care devices to prepare stem cells from adipose tissue for use in the same surgical procedure in the same operating room. These products are exempted from the Directive of Tissues and Cells, and because they are not intended to be placed on the market, Dr. Kleinhenz advocated that in this specific situation it is not clear if the ATMP regulation should be applied. Although the CAT acknowledged the complexity of the regulation for these new emerging products, its current view is that if the products (tissues or cells) are either substantially manipulated or are intended for non-homologous use, they fall under the provision of ATMP regulation whether or not they are manipulated in the same surgical procedure. There are also specific provisions such as hospital exemption for ATMP produced for a single use in a specific patient.

This discussion highlighted the importance of explaining to the stakeholders the CAT's current criteria for ATMP classification: these have been reflected in the CAT reflection paper on ATMP classification [4], which was presented by Dr. Ferry. From the experience gained over the past 5 years, the CAT has refined its criteria for classification and a revision of the reflection paper is in progress, with a particular focus on the CAT position on substantial manipulation and non-homologous use. In his presentation, Dr. Ferry presented this CAT guidance paper that provides the basic tools for ATMP classification. He highlighted with some specific examples how to discriminate between ATMPs and non-ATMPs in difficult situations, e.g., discrimination between gene therapy products belonging to ATMPs and gene therapy products considered as vaccine against infectious diseases (which are per the definition of a GTMP not considered ATMPs). More importantly, he discussed the definition of substantial manipulation, emphasising that the legislation only laid down the manipulations considered as non-substantial. The issue of whether or not enzymatic treatment could be considered as non-substantial manipulation was also discussed with the audience. The present CAT position reflected in the revised reflection paper is that when enzymatic treatment is aimed at isolating or separating cells, it is a substantial manipulation. In general, enzymatic digestion of tissues leads to a cell suspension where the cell structure (e.g., surface molecules) and functionality are altered when compared to the situation in an intact tissue. One exception is the collagenase treatment used for procurement of isolated pancreatic islets. Indeed, in this specific situation single beta cells are not isolated and an intact epithelium structure of the islet is maintained. In this instance beta cells are not considered substantially manipulated. The definition of homologous versus non-homologous use was also presented. It was indicated that for the CAT homologous use (same essential func- 
tion) is restricted either to cells maintaining the original function in the same anatomical or histological environment, or for transplantation of a tissue to another location in the same anatomical or histological environment to achieve the same essential function. As bone marrow is regarded as a tissue, use of bone marrow cells for another purpose than haematopoietic reconstitution is considered non-homologous use.

The CAT members were not able to answer all specific questions from the audience related to ATMP classifications and they warmly welcomed the audience to submit comments on the revised CAT reflection paper on classification. ATMP developers are advised to submit an ATMP classification request to the CAT, especially for product at the border between ATMPs and cell-tissue preparations / transplants

\section{Cell-Based Therapies for Cardiac Repair}

In the third section of the workshop, CAT members and an invited expert presented information and considerations on cellbased therapies for cardiac repair, which were discussed in a CAT Workshop held in May 2014.

Despite increasing clinical research activity aiming to show that stem cells have the potential to regenerate the human heart, the final answer is still awaited. The developments are challenging, and data from first confirmatory, large clinical trials are expected to guide the field further in the development. Dr. Schüßler-Lenz gave an introduction to the field and presented the challenges and opportunities currently faced by the developers in this field. The first clinical studies were exploratory trials, using autologous unselected mononuclear cells (MNCs) either from bone marrow or peripheral blood. Since then an increased diversity has been observed regarding cell source as well as degree of manipulation of cells, towards cell selection, culturing and use of allogeneic cells. Clinical studies are conducted in different cardiac diseases, like acute myocardial infarction (MI) and chronic heart failure. More emphasis is placed on understanding the mode of action of the cells in relation to the condition to be treated. A number of randomised placebo-controlled phase III trials are being conducted in the EU, and prove of efficacy and safety is awaited.

Dr. Assmus gave a brief overview of the clinical development of bone marrow-derived mononuclear cell (BMC) therapy for myocardial repair, with the focus on MI. The first clinical studies were exploratory trials, using autologous unselected MNCs either from bone marrow or peripheral blood. Since then an increased diversity has been observed regarding cell source as well as degree of manipulation of cells, towards cell selection, culturing and use of allogeneic cells. Since the first clinical studies, new products based on novel concepts have emerged. More emphasis has been placed on understanding the mode of action of the cells in the condition to be treated, which has led to different treatment approaches. A number of randomised placebo-controlled phase III trials have been conducted in the EU. According to Dr. Assmus, regenerative therapies have now emerged as a promising novel approach to improve heart function and prevent the development of end-stage heart failure. In patients with acute MI, recent metaanalyses suggested a moderate but sustained enhancement of left ventricular (LV) function and improved clinical outcome following administration of BMCs [13]. Dr. Assmus highlighted that identifying the characteristics of patients who will ultimately benefit from cell therapy has proven to be essential, and it was recently demonstrated that baseline LV ejection fraction (LVEF) determines long-term effect BMCs in ST-segment elevation myocardial infarction (STEMI) [14]. Furthermore, it was emphasised that several factors are influencing the function of autologous BMCs, such as intrinsic and extrinsic factors of the cells, storage and functionality. Dr. Assmus concluded that cell therapy with autologous BMCs is a realistic option in patients with large acute myocardial infarction, and consequently the largest trial, BAMI (supported by EU funding FP7), started in 2013. This is a randomised, controlled multi-centre study conducted in Europe with an estimated enrolment of 3,000 patients, aiming to find the effect of intracoronary reinfusion of BMCs on all-cause mortality in STEMI. Dr. Assmus also briefly mentioned that in patients with chronic post infarction heart failure, BMC therapy has demonstrated heterogeneous results so far and generally seems to have minor efficacy. Enhancement strategies are under way, but more basic research remains to be done.

Dr. Salmikangas presented the multiple approaches that have been used so far, including simple non-manipulated cell preparations from peripheral blood or bone marrow and advancing to use of mesenchymal stem cells (MSCs), skeletal myoblasts or even isolated, cultured cardiomyocytes. The challenges from a quality/ manufacturing perspective relating to characterisation of such products were discussed, as the experience has shown that in cases where the product is not characterised and its quality not defined, non-clinical and clinical results may not be comparable and will be difficult to repeat. Furthermore, a product target profile is always required for clinical studies to ensure patient safety. It was also discussed why results from early clinical studies have shown moderate or inconclusive results, and questions were raised whether this could be due to variability at the product level.

There was also discussion on cell origin/donation, including donor medication and other aspects that could have an impact on the final product. Manufacturing and new technologies were also addressed, as well as impact of cell separation techniques on quality of the final product. Products requiring in vitro cell culture pose additional concerns, as well as differentiation of proliferating cells towards intended cell populations (MSCs, cardiac cells). Especially challenging appears to be how to demonstrate functionality/potency and correct phenotype/genotype of the various cell products for the intended use. Potency testing should ideally follow mode of action, and it was acknowledged that different assays might be required for sorted and cultured cells versus BMCs / blood-derived MNCs. For different product types variable mode of actions have been proposed, the first claims being that MNCs or MSCs would form new cardiac tissue changing later to claims of paracrine effects. For quality control, the analytical methods play a key role, 
and new emerging techniques may provide solutions to current constraints.

Dr. Beuneu highlighted that the crucial non-clinical issues in current CBMP development for cardiac repair are connected to selection of appropriate animal models and study design to determine pharmacodynamic and pharmacokinetic properties, like biodistribution/migration and persistence of the cells in the body, to define a dose and explore potential toxicity. At present, there are no standardised animal models for non-clinical studies of CBMPs for cardiac repair. Large and small animals are used for the purpose of non-clinical testing, both with advantages and disadvantages as well. The capacity of non-clinical studies to predict human clinical outcome has been challenged by some authors $[15,16]$. However, Dr. Beuneu concluded that there is clear evidence that appropriate animal studies are relevant for translational purposes [17], and they are mandatory to assess the risks of a new CBMP and predict its feasibility, safety and efficacy.

The final speaker in this session was Dr. Doevendans who focused on the clinical aspects. He highlighted the important variations in dose, application route, timing and cell type used. Based on the outcome of the larger clinical trials and supported by a Cochrane analysis that included 1,700 patients from 33 different trials, several conclusions can be drawn [18]. Application of BMCs through various routes post $\mathrm{MI}$ and in patients with cardiac failure appears not to raise safety concerns. There is a tendency to a larger effect if cells are delivered 5-7 days post MI [19]. In addition the assumption is confirmed that the best results are obtained in patients with a poor LV function. However, if MRI imaging for the assessment of LV function is considered to be the gold standard, no effect of cell therapy is measured overall after 6 months and a very modest non-significant effect after 12 months. Disregarding the imaging modality selected and including all patients, a recovery of LVEF was reported of $2.9 \%$. In addition, a positive relation was observed between dosage and improvement of LV function (up to $5.6 \%$ with $>10^{10}$ cells). No effect on mortality was reported, undermining the primary outcome chosen in the BAMI trial. The cardiac stem cell field was questioned in a recent paper indicating a linear relationship with the number of errors or missing data in the clinical study report and the effect size [20]. Dr. Doevendans concluded that lack of standardisation in products and protocols and paucity of in vitro functional assessments is blurring the outlook for the clinical stem cell field. There is a clear role of academias, SMEs and regulatory bodies to suggest and implement harmonising guidelines to bring the field forward.

\section{Disclaimer}

The views expressed in this article are the personal views of the authors, and may not be understood or quoted as being made on behalf of the EMA or the CAT or reflecting the position of the CAT.

\section{Disclosure Statement}

The authors have no conflicts of interest to declare in relation to the aspects addressed in this article.

\section{References}

1 The European Parliament and the Council of the European Union: Regulation (EC) No. 1394/2007 of the European Parliament and the Council of 13 November 2007 on Advanced Therapy Medicinal Products and amending Directive 2001/83/EC and Regulation (EC) No 726/2004. Official Journal of the European Union 10.12.2007;L324:121-137.

2 European Medicines Agency: CAT: Agendas, minutes and reports. www.ema.europa.eu/ema/index.jpp?curl= pages/news_and_events/document_listing/document_listing_000196.jspermid $=$ WC0b01ac05800292a8\#section3.

3 Maciulatis R, D’Apote L, Buchanan A, Pioppo L, Schneider C K: Clinical development of advanced therapy medicinal products in Europe: evidence that regulators must be proactive. Mol Ther 2012;20:479-482.

4 European Medicines Agency: Draft reflection paper on classification of advanced therapy medicinal products (EMA/CAT/600280/2010 Rev.1).www.ema.europa.eu/ ema/doc_index.jsp?curl=pages/includes/document/ document_detail.jsp?webContentId $=$ WC500169466 $~ \mathrm{~m}$ url=menus/document_library/document_library. jspermid=0b01ac058009a3dc.

5 The European Parliament and the Council of the European Union: Directive 2004/23/EC of the European Parliament and of the Council of 31 March 2004 on setting standards of quality and safety for the donation, procurement, testing, processing, preservation, storage and distribution of human tissues and cells. Official Journal of the European Union 7.1.2004;L102:48-58.
6 The Commission of the European Communities: Commission Directive 2009/120/EC of 14 September 2009 amending Directive 2001/83/EC of the European Parliament and of the Council on the Community code relating to medicinal products for human use as regards advanced therapy medicinal products. Official Journal of the European Union 15.9.2009;:L242:3-12.

7 European Pharmacopoeia. 5.2.12 Raw materials for the production of cell-based and gene therapy medicinal products (PA/PH/Exp. RCG/T (14) 5 ANP). Pharmeuropa 2014, 26.4 (October 1, 2014)

8 European Medicines Agency: Guideline on risk-based approach according to Annex I, Part IV of Directive 2001/83/EC applied to Advanced Therapy Medicinal Products (EMA/CAT/CPWP/686637/2011). www.ema. europa.eu/docs/en_GB/document_library/Scientific_ guideline/2013/03/WC500139748.pdf.

9 The Commission of the European Communities, Directorate General Health and Consumers. Summary of the responses to the public consultation on the Regulation on Advanced Therapies. May 22, 2013. http:// ec.europa.eu/health/files/advtherapies/2013_05_pc_ atmp/2013_04_03_pc_summary.pdf.

10 The Commission of the European Communities, Directorate General Health and Consumers. Report on the application of Regulation (EC) No 1394/2007 on Advanced Therapy Medicinal Product. 1 April 2014. http://ec.europa.eu/health/files/advtherapies/2014_ atmplatmp_en.pdf.
11 European Medicines Agency: Summaries of scientific recommendations on classification of advanced therapy medicinal products. www.ema.europa.eu/ema/ index.jsp?curl=pages/regulation/general/general_content_ 000301.jspermid =WC0b01ac05800862c0

12 European Medicines Agency: Innovation Task Force. www.ema.europa.eu/ema/index.jsp?curl=pages/ regulation/general/general_content_000334.jspermid= WC0b01ac05800ba1d9.

13 Jeevanantham V, Bulter M, Saad A, Abdel-Latif A, Zuba-Surma EK, Dawn B: Adult bone marrow cell therapy improves survival and long-term improvement in cardiac parameters: a systematic review and meta-analysis. Circulation 2012;126 (5): 551-568.

14 Assmus B, Leistner DM, Schächinger V, Erbs S, Elsässer A, Haberbosch W, Hambrecht R, Sedding D, Yu J, Corti R, Mathey DG, Barth C, Mayer-Wehrstein C, Burck I, Sueselbeck T, Dill T, Hamm CW, Tonn T, Dimmeler S, Zeiher AM; REPAIR-AMI Study Group: Long-term clinical outcome after intracoronary application of bone marrow-derived mononuclear cells for acute myocardial infarction: migratory capacity of administered cells determines event-free survival. Eur Heart J 2014;35:1275-1283.

15 Lafont A, Faxon D: Why do animal models of post-angioplasty restenosis sometimes poorly predict the outcome of clinical trials? Cardiovasc Res 1998;39:50-59.

16 Rosenzweig A: Cardiac cell therapy - mixed results from mixed cells. N. Engl J Med 2006;355:1274-1277. 
17 van der Spoel TI, Jansen of Lorkeers SJ, Agostoni P, van Belle E, Gyöngyösi M, Sluijter JP, Cramer MJ, Doevendans PA, Chamuleau SA: Human relevance of preclinical studies in stem cell therapy: systematic review and meta-analysis of large animal models of ischaemic heart disease. Cardiovasc Res 2011;91:649-658.
Clifford DM1, Fisher SA, Brunskill SJ, Doree C, Mathur A, Watt S, Martin-Rendon E: Stem cell treatment for acute myocardial infarction. Cochrane Database Syst Rev 2012;2:CD006536.

19 Simari RD, Pepine CJ, Traverse JH, Henry TD, Bolli R, Spoon DB, Yeh E, Hare JM, Schulman IH, Anderson RD, Lambert C, Sayre SL, Taylor DA, Ebert RF, Moyé LA: Bone marrow mononuclear cell therapy for acute myocardial infarction: a perspective from the Cardiovascular Cell Therapy Research Network. Circ Res 2014;114:1564-1568.
20 Nowbar AN, Mielewczik M, Karavassilis M, Dehbi HM, Shun-Shin MJ, Jones S, Howard JP, Cole GD, Francis DP; DAMASCENE writing group: Discrepancies in autologous bone marrow stem cell trials and enhancement of ejection fraction (DAMASCENE): weighted regression and meta-analysis. BMJ 2014; 348:g2688. 